\title{
Efectos negativos de la radiación ionizante empleada en diagnóstico odontológico
}

\author{
Negative effects of ionizing radiation used in dental diagnosis
}

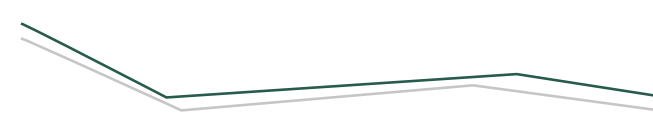

Deyanira Serrato*, Renato Nieło-Aguilar*凶, Asdrúbal Aguilera-Méndez***

Serrato, D., Nieto-Aguilar, R., \& Aguilera-Méndez, A. (2018). Efectos negativos de la radiación ionizante empleada en diagnóstico odontológico. Investigación y Ciencia de la Universidad Autónoma de Aguascalientes, 26(74), 81-87.

\section{RESUMEN}

La labilidad del componente celular humano, bajo efecto de radiación ionizante (RI), involucra cambios alterados celulares y subcelulares en el organismo, sobre todo en células en reproducción continua y menor grado de diferenciación. El potencial carcinogénico, mutagénico y genotóxico de la RI es explicado en esta revisión. Se clarifican los efectos de la exposición a RI a dosis inadecuadas en el área odontológica, y a nivel molecular, celular y orgánico; los mecanismos que accionan el posible daño, las alternativas para el control de absorción, la justificación para su uso como medio de diagnóstico dental a nivel pediátrico y adulto, y las tecnologías emisoras de radiación en odontología. La naturaleza nociva pero necesaria de la radiación en el medio dental podría concientizar a instituciones de salud dental, a su personal y a los pacientes involucrados, a cumplir y hacer cumplir las medidas de control

Palabras clave: radiación ionizante; radio sensibilización; radiología dental; rayos $X$.

Keywords: ionizing radiation; radio sensitization; dental radiology; X-rays.

Recibido: 16 de agosto de 2017, aceptado: 14 de marzo de 2018

* Facultad de Odontología, División de Estudios de Posgrado e Investigación, Universidad Michoacana de San Nicolás de Hidalgo. Av. San Juanito Itzícuaro km. 5, Colonia Arboledas Valladolid, C. P. 58330, Morelia, Michoacán, México. Correo electrónico: gelato.serrato@ yahoo.com.mx; nieto.renato@gmail.com

** Instituto de Investigaciones Químico Biológicas, Universidad Michoacana de San Nicolás de Hidalgo. Av. Francisco J. Múgica s/n, Edificio B-1 planta baja, Colonia Felícitas del Río, Ciudad Universitaria, C. P. 58030, Morelia, Michoacán, México. Correo electrónico: asdruaguilera@yahoo.com.mx

$凶$

Autor para correspondencia durante procedimientos clínicos dentales que la emplean.

ABSTRACT

The lability of the human cellular component, under the effect of ionizing radiation (IR), involves altered cellular and sub-cellular changes in the organism, especially in cells in continuous reproduction, and lower degree of differentiation. The carcinogenic, mutagenic and genotoxic potential of IR is explained in this review. The effects of exposure to IR are clarified, when inadequate doses are exposed in dentistry at the molecular, cellular and organic levels. Also explains the mechanisms which trigger possible damage, alternatives for the control of absorption, and the validation for its use as a means of dental diagnosis at pediatric and adult stages, and finally, it exposes radiation emitting technologies in dentistry. The harmful but necessary nature of the radiation at the dental environment, could raise awareness of dental health institutions, their staff and involved patients, to comply with and to enforce control measures during dental clinical procedures which use IR.

INTRODUCCIÓN

\section{Radiación en general}

La radiación consiste en la emisión, propagación y transferencia de energía de algún medio a otro, en forma de ondas o partículas del campo electromagnético. En el ser humano, esta energía en forma de ondas se absorbe durante toda la vida, y es de tipo acumulativo. La exposición y absorción a la energía radiante puede ser cuantificada en niveles, lo que ocasionará o no, para determinado nivel, cambios 
altamente perceptibles en las funciones orgánicas. Básicamente, el nivel depende directamente de la frecuencia, que como regla general presenta que cuanto más alta es (longitudes de onda más cortas) posee mayor poder de penetración.

Las fuentes de radiación se dividen principalmente en las de tipo no ionizante y las ionizantes. Según la OMS (Organización Mundial de la Salud), las primeras son radiaciones compuestas por cuantos de luz sin energía suficiente para romper los enlaces moleculares. Por otro lado, las segundas desprenden ondas electromagnéticas que transportan tanta energía por cuanto de luz, que son capaces de romper los enlaces entre las moléculas. Algunos ejemplos de la radiación no ionizante incluyen a las ondas de radio, microondas, rayos infrarrojos, luz visible, electricidad e inclusive una pequeña parte de los rayos ultravioleta. Las radiaciones ionizantes en su caso involucran a los rayos gamma que emiten los materiales radioactivos, los rayos cósmicos y los rayos $X$ (figuras 1 y 2 ).

De las radiaciones emitidas dentro de la naturaleza se encuentran, entre otras, la radiación cósmica, así como la radioactividad debajo de la tierra. Por otro lado, las radiaciones artificiales comprenden desde las generadas en los relojes simples, en antenas, incluso en aparatos de televisión, y desde las utilizados más frecuentemente de manera actual: las pantallas de computadoras y teléfonos ceIulares (Hayflick \& Moorhead, 1961; Jacobson, 1952; Shao, Luo, \& Zhou, 2014). La radiación que emite este hardware tecnológico ha demostrado ser perjudicial en mayor o menor medida, que es directamente proporcional al tipo de fuente generadora. En este contexto, el ser humano recibe a lo largo de su existencia radiación artificial ionizante por fuentes generadoras de rayos $X$, que es necesaria para el diagnóstico médico y dental por imagen.

Dada la importancia del empleo de dicha radiación en el área dental y debido a que ha permitido la visibilidad de estructuras y ultraestructuras anatómicas densas, su empleo ha beneficiado al conocimiento y entendimiento patológico de tejidos no visibles por inspección simple. Sin embargo, y debido a que este tipo radiación (RI) puede causar alteraciones orgánicas en diferente grado, la presente revisión tiene como objetivo exponer su estado actual en el área odontológica, precisando el mecanismo de acción sobre órganos, tejidos, células y componentes celulares; su efecto nocivo so- bre los mismos y, por último, evidencia las directrices para su empleo más apropiado en técnicas radiográficas bucodentales. Los criterios de inclusión de esta revisión contemplaron estudios aleatorios, que pretendían conocer los efectos de la exposición a la RI en odontología. La búsqueda se realizó en la plataforma de datos de PUBMED, sin límite de periodo de tiempo y se utilizaron las palabras clave: ionizing radiation, radio sensitization, dental radiology, X-rays.

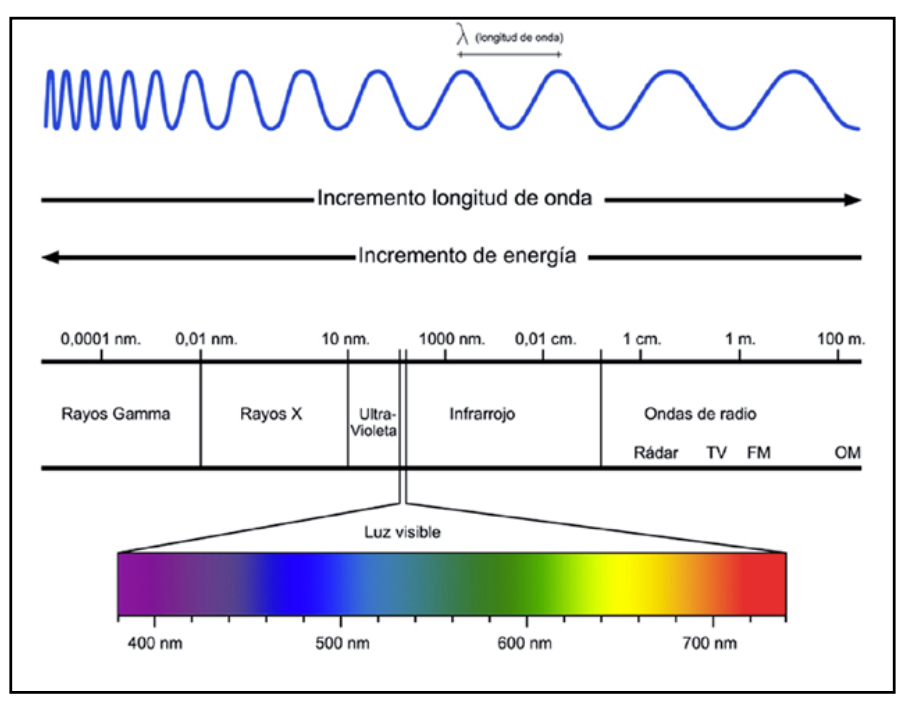

Figura 1. Esquema que muestra la relación inversa entre el incremento de la longitud de onda y el incremento de energía en el espectro electromagnético. Las ondas electromagnéticas de baja frecuencia se denominan campos electromagnéticos, y las de muy alta frecuencia radiaciones electromagnéticas. Según sea su frecuencia y energía, las ondas electromagnéticas pueden clasificarse en radiaciones ionizantes o radiaciones no ionizantes.

Imagen tomada de Blog Termografía (2015).

\section{Mecanismo de la radiación ionizante $X$ sobre proteínas y las matrices intra y extracelulares}

En el caso de la radiación por rayos $X$ la acción de sus fotones puede generar en el componente tisular cambios anormales en sus elementos químicos, como es el caso particular del agua localizada dentro y fuera de la célula, que puede ser convertida en dioxidano. Este compuesto ha sido considerado un veneno celular; sin embargo, la propia homeostasis celular lo inactiva bajo ciertos grados radiantes moderados y leves. La energía radiante puede afectar la estructura molecular de una amplia gama de proteínas incluyendo: enzimas, inhibidores, hormonas, etc. Dicha alteración ocasiona la ineficacia en la función precisa de cada una de ellas, lo que derivada del desequilibrio parcial 0 total molecular (Hall \& Giaccia, 2006). De esta 


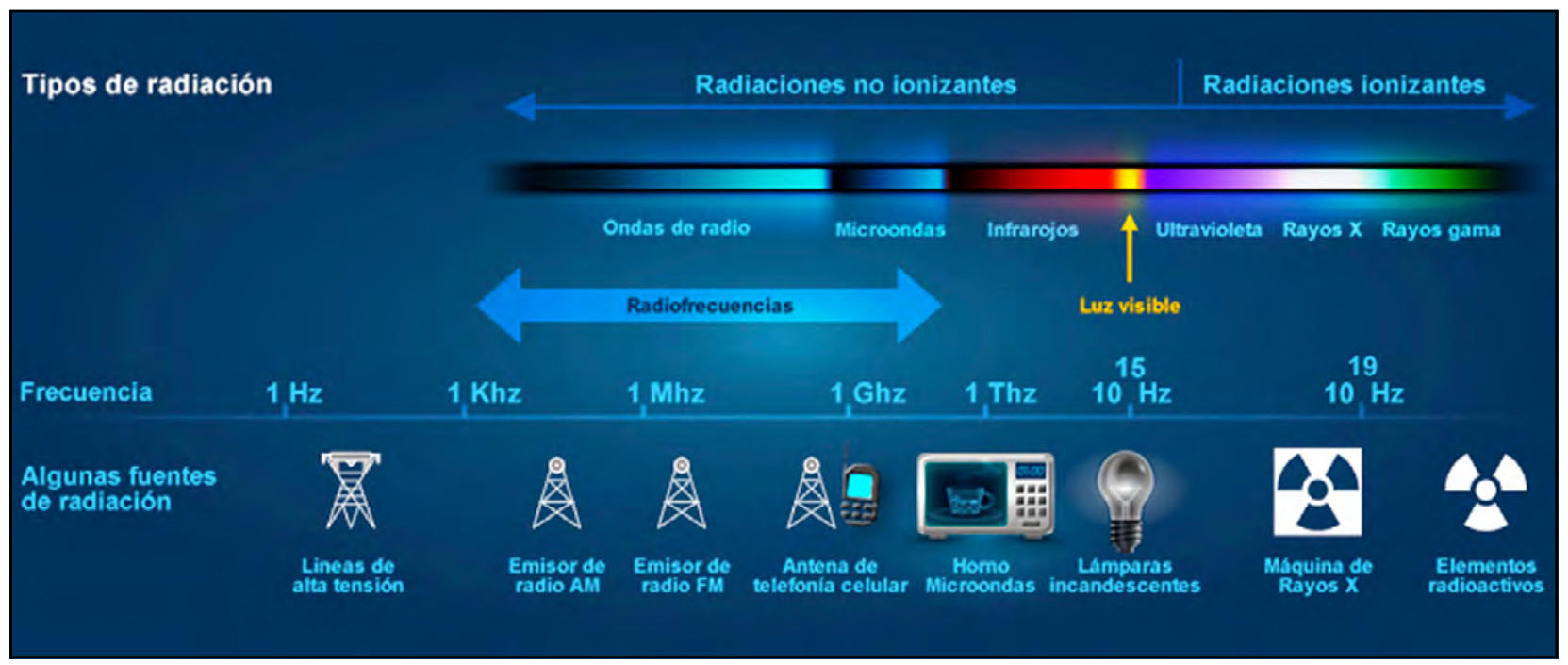

Figura 2. Esquema ilustrativo de fuentes de radiación conocidas y su relación con la frecuencia en el campo electromagnético.

Imagen tomada de Organización Mundial de la Salud (s. f.).

manera, cualesquier tipo de frecuencia radiante, aun escasa, produce cambios importantes en las estructuras de las células y de la matriz extracelular, que derivan de señalizaciones alteradas dentro y fuera de las células. En este tenor, para las células menos diferenciadas las alteraciones pueden ser más fáciles de ocurrir, ya que son las más radiosensibles (Buonanno, De Toledo, \& Azzam, 2011).

\section{Radiación ionizante y cáncer}

La radiación ionizante es bien conocida como un agente carcinógeno para los seres humanos. Las alteraciones cromosómicas y la formación de micronúcleos en el citoplasma de la célula son evidencia biológica precoz de la carcinogénesis (Waingade \& Medikeri, 2012). La variación en la capacidad de reparación en los fragmentos de información genética ante las radiaciones puede influir en la capacidad de reparación ultraestructural del ADN, lo que origina riesgos para generar cáncer (Cho et al., 2009). Cuando es el caso, las vías principales de reparación del ADN implican a varios mecanismos: el primero involucra a la reparación por escisión (BER) (Svilar, Goellner, Almeida, \& Sobol, 2011; Wilson, Kim, Berquist, \& Sigurdson, 2011); el segundo, a la reparación por escisión de nucleótidos (NER) (Vermeulen, 2011); el tercero, al desajuste de reparación (Jiricny, 2006); el cuarto, a la unión de extremos no homólogos (NHEJ) y, por último, el correspondiente a la reparación de recombinación homóloga (HRR) (Holthausen, Wyman, \& Kanaar, 2010; Kass \& Jasin, 2010).
Sin embargo, no todas las células presentan eficiencia para autorrepararse, y menos en acumulación de altas dosis de radiación, incluso en las células con un grado de diferenciación alto (Adams, Hawkins, Povirk, \& Valerie, 2010; Fung \& Weinstock, 2011; Luo et al., 2012; Maynard et al., 2008). De hecho, las células en cultivo han mostrado ser radiosensibles en diferentes grados. Algunos efectos importantes reportados en estas células incluyen, en primera instancia, daño al código genético, merma en la capacidad de reproductiva de la célula y, por último, su muerte. Estos eventos involucran de forma final una alteración directa sobre las futuras copias de la célula (figura 3).

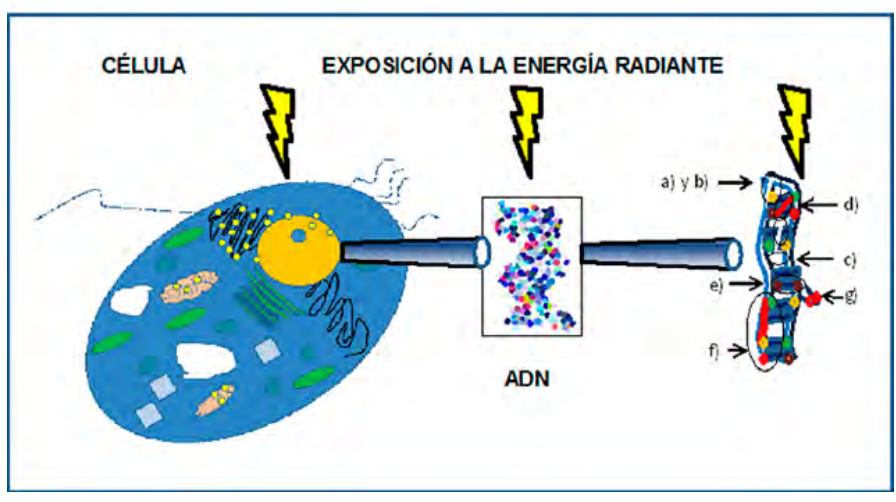

Figura 3. Lesiones en ADN inducidas por RI: a) rompimiento de puentes de hidrógeno; b) rompimiento de la cadena helicoidal única o doble; c) pérdida de bases; d) uniones cruzadas ADNADN; e) cambio de bases; f) uniones cruzadas entre proteína y ADN; g) dímero de bases pirimidínicas.

Figura elaborada por Renato Nieto-Aguilar. 
IIVESTIGACIÓn Y CIERCIA DE LA UחIVERSIDAD AUTÓOOOMA
Radiación ionizante para diagnóstico en centros de salud

El uso de radiación ionizante a través de rayos $X$ para el diagnóstico por imagen en los sistemas de salud se ha incrementado y perfeccionado en los últimos años. Ello incluye bajas exposiciones a radiación con análisis más precisos e imágenes nítidas, conversiones de imagen en caso necesario, y mediciones con software digital integrado a la imagen radiográfica digital. Sin embargo, en casos específicos, algunos pacientes requieren de un número alto de exposiciones o estudios radiográficos a frecuencias más altas. Desafortunadamente, en estos casos el factor riesgo puede incrementarse, lo que a su vez puede involucrar efectos adversos sobre los tejidos expuestos. Entre otros, se incluye el carcinoma, tanto en pacientes como en personal clínico (Lee et al., 2016; Praveen, Shubhasini, Bhanushree, Sumsum, \& Sushma, 2013; White \& Mallya, 2012; White \& Pharoah, 2009). A este respecto, Memon, Godward, Williams, Siddique y Al-Saleh (2010) llevaron a cabo un estudio acerca del riesgo de cáncer de tiroides derivado de una exposición con radiación ionizante por rayos $X$.

El estudio fue debatido ampliamente, y se concluyó que la optimización de la dosis en la radiografía dental debe ser abordada con urgencia. Las evidencias existentes de respuesta, que presentan las células de estirpes bucodentales, como las células troncales del ligamento periodontal, y pulpa aisladas y cultivadas bajo radiación ionizante (de 2 a 20Gy) mostraron alteraciones en la estructura de su ADN, lo que redundó en alteraciones del ciclo celular (Cmielova et al., 2013). Derivada de lo anterior es la tecnología que maneja radiación ionizante, la que a su vez puede controlar en parte ciertos efectos adversos de la Rl. Por ello dicha tecnología será expuesta a continuación de manera amplia.

\section{Tecnologías en radiación ionizante aplicadas a odontología}

La tecnología utilizada en las ciencias dentales para el aprovechamiento de la radiación ionizante ha mostrado un desarrollo impresionante en los últimos años, gracias a la aplicación digital sobre la imagen radiográfica por los rayos $X$. Sin embargo, al momento actual aún existen contradicciones acerca del tipo de radiografía a utilizar, al momento de llevar a cabo un diagnóstico. Algunos investigadores coinciden en que la radiografía panorámica debe utilizarse ampliamente para complementar el examen clínico, ya que es menos perjudicial que la realización de varias radiografías de tipo periapical (Cerqueira et al., 2004; White \& Pharoah, 2004).

Sin embargo, en un estudio realizado por Waingade y Medikeri (2012) se demostró que la radiografía panorámica puede inducir efectos genotóxicos en células epiteliales bucales y, por tanto, se debe tener en cuenta este riesgo, al extremar precauciones. En un estudio realizado en una población india se evidenció que incluso los niveles bajos de exposición a la radiación asociada con la radiografía panorámica puede ocasionar cambios genéticos en las células que se exponen a este tipo de radiación, independientemente del tiempo y de la dosis a la cual son expuestas (Waingade \& Medikeri, 2012).

Otros investigadores mencionan que la dosis total de exposición con la tomografía de haz cónico dental computarizada (CBCT), relativamente más baja que la de la tomografía computarizada, sigue siendo considerablemente más alta que la correspondiente a la radiografía dental convencional (Lee et al., 2016; Loubele et al., 2009; Roberts, Drage, Davies, \& Thomas, 2009). Más aun, se reporta que es preciso conocer los diferentes modelos de CBCT al tomar diferentes campos de visión (FOV), debido a que la dosis de radiación variará entre ellos. Roberts et al. (2009) midieron la dosis absorbida de tres diferentes modelos de CBCT, a completo campo de visión. El resultado fue sorprendentemente variable, ya que involucró de 2,5 mGy a 32,7 mGy; por tanto, la evaluación de dosis de radiación en la odontología se ha convertido en un verdadero reto para científicos y tecnólogos, que han manifestado la necesidad de reducir las dosis de radiación en el diagnóstico por imagen.

\section{Reducción y control de radiación ionizante en pro- cedimientos odontológicos}

Los principios de la protección radiológica sugeridos por la Comisión Internacional de Protección Radiológica (ICRP) consisten en la justificación, optimización y limitación de la dosis individual. En virtud de estos principios la limitación de la dosis en la medicina no está especificada por estas regulaciones (Hart, Hillier, \& Wall, 2009). Para el caso de odontología tampoco existe alguna limitación en muchos países, que sin embargo es requerida. En 2006 la Organización Internacional de Energía Atómica (OIEA) puso en marcha un proyecto de una tarjeta inteligente, destinada a desarrollar un sistema para el seguimiento de la dosis de radiación en cada individuo. La reunión de la OIEA en 2013 indicó que el 


\section{IIVESTIGAGIÓn Y CUERCIA DE LA UกIVERSIDAD AUTÓOOOMA DE RGUASCALIERTES}

seguimiento de la dosis de cada paciente con una tarjeta inteligente se ha implementado en Estonia, Finlandia, Malta y Suecia.

Por otro lado, Argelia, Bulgaria, Egipto, Macedonia, Países Bajos, Rumania, Corea del Sur y España están trabajando actualmente en la construcción de un sistema de dicho tipo, que desafortunadamente no ha mostrado el avance obtenido hasta el momento actual (Henriques, 2013). Es de resaltar que un estudio que evaluaba la radiación emitida por 79 unidades de rayos $X$, utilizada en clínicas dentales de Varsovia con control de radiación, encontró que $30.4 \%$ de los pacientes recibe hasta 10 veces más radiación ionizante, lo que sobrepasa la referencia emitida por la legislación polaca (Bekas \& Pachocki, 2013; Rehani y Berris, 2013). Asimismo, encuestaron a 40 países en cuanto a la disponibilidad de fijar un número de identificación para el paciente con un sistema de seguimiento de dosis de radiación. En 2015 Rehani cristaliza dicha propuesta informando de la disponibilidad de un sistema de identificación del paciente (ID) interconectado a todos los hospitales, que haría posible la construcción de un sistema de seguimiento de dosis para todo el mundo.

\section{Justificación de dosis de radiación sobre el ser hu- mano}

El control de la RI en los gabinetes odontológicos y en las clínicas de atención dental podría ser factible al reducir de forma efectiva el número de estudios radiográficos, la película a utilizarse, los reactivos reveladores, la energía disponible para el análisis

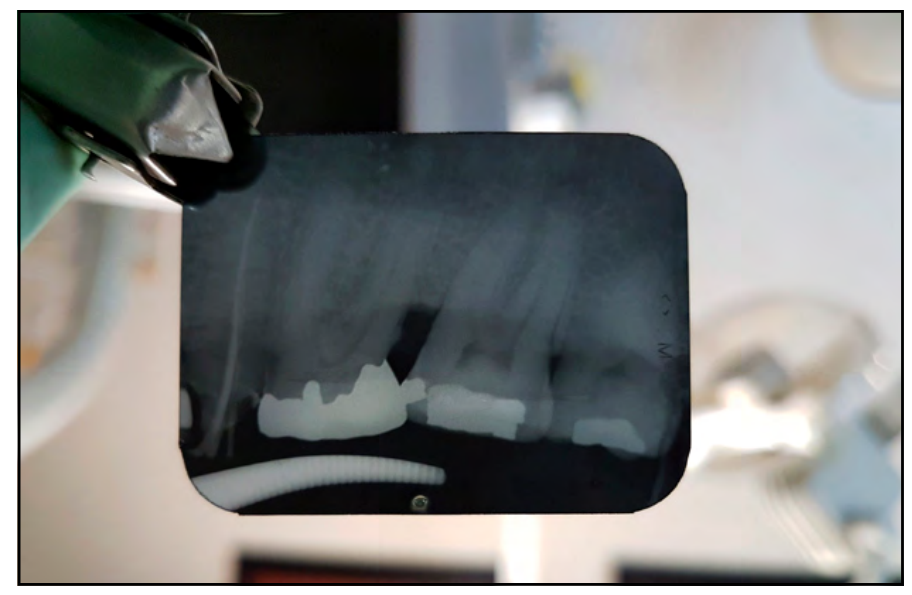

Figura 4. Fotografía de una radiografía dental periapical con claridad y nitidez. Se controlaron los factores que pueden ser negativos al paciente por la radiación ionizante, que desprende el aparato de rayos $X$.

Fotografía tomada por el equipo de investigación. radiográfico y la dosis de una exposición de radiación baja con una alta definición de análisis digital, que permita incluso a las dosis bajas incrementar la calidad de la imagen (Hellén-Halme \& Nilsson, 2013; Shin et al., 2014). Estos aspectos deben individualizarse (figura 4) con respecto a las necesidades únicas de cada paciente $y$, por tanto, la precisión de una historia clínica detallada y un examen clínico preciso se consideran la base de la encomienda (Kim \& Mupparapu, 2009). A este respecto se sugiere tomar medidas precautorias específicas para la exposición de la radiación sobre pacientes pediátricos, ya que para ellos representa un riesgo potencial mayor que para los adultos.

Las medidas precautorias de exposición a la RI en pacientes de menor masa corporal se acotan a continuación.

$$
D=E \text { Absorbida }
$$

$m$

\section{$D=$ dosis absorbida}

E absorbida= energía promedio absorbida por el medio $m=$ masa del medio que absorbió la energía (Medina Gironzini, 2014).

La explicación a la fórmula de la <energía promedio absorbida por unidad de masa del medio absorbedor> pone de manifiesto que a un menor tamaño corporal traducido generalmente a una masa corporal menor (como es el caso de los pacientes pediátricos) la dosis de radiación es mayor que en la de un adulto. Con lo anterior se han tomado medidas precautorias específicas para la exposición de bebés y niños a rayos $X$. Las mismas incluyen blindaje de protección, como el uso de protectores a base de plomo. Por ejemplo: mandiles y protectores para cuello de plomo, colimadores en el aparato propiamente dicho, filtros para aumento de voltaje con disminución de tiempos de exposición, control automático de exposición y capacitación del personal que realiza la exposición para optimizar las condiciones del área operatoria. Se incluyen técnicas de inmovilización para la zona que recibe la exposición de tipo no agresivo, técnicas de intensificación de imagen como la utilización de chasis plástico y colimadores externos adecuados al caso (International Atomic Energy Agency, 2013; Kohn et al., 1996). 
La exposición a la radiación se acumula en las células de los niños a lo largo de su vida y cualquier falla o escasa consciencia podría derivar en dosis de radiación más altas de lo necesario, si los ajustes de exposición no son considerados en relación con el tamaño corporal pequeño y madurez tisular (SEDENTEXCT Guideline Development Panel, 2012; Shin et al., 2014). Se ha reportado que el riesgo de exposición a RI en pacientes menores de 10 años es tres veces mayor que a la edad de 30 años (SEDENTEXCT Guideline Development Panel, 2012). Por tanto, ha sido justificable la solicitud de las organizaciones e institutos incluyentes sobre el control de la RI que debería evitarse sobre cualquier ser humano y de cualquier edad o condición (American College Of Radiology, s. f.; Sonawane, Sunil Kumar, Singh, \& Pradhan, 2011).

\section{REFERENCIAS}

- Adams, B. R., Hawkins, A. J., Povirk, L. F., \& Valerie, K. (2010). ATM-independent, high-fidelity nonhomologous end joining predominates in human embryonic stem cells. Aging (Albany NY), 2(9), 582-596.

- Aliada contra el cáncer. (García B.). (2013). Recuperado de https://es.slideshare.net

- American College of Radiology. (s. f.). ACR appropriateness criteria, 2013 [Portal electrónico]. Recuperado de http:// www.acr.org/Quality-Safety/Appropriateness-Criteria

- Bekas, M., \& Pachocki, K. A. (2013). The dose received by patients during dental X-ray examination and the technical condition of radiological equipment. Medycyna Pracy, 64 (6), 755-759.

- Blog Termografía. (3 de noviembre de 2015). Qué es el espectro electromagnético [Imagen ilustrativa de entrada de blog]. Recuperada de http://blogtermografia.com/quees-el-espectro-electromagnetico/

- Buonanno, M., De Toledo, S. M., \& Azzam, E. I. (2011). Increased frequency of spontaneous neoplastic transformation in progeny of bystander cells from cultures exposed to denselyionizing radiation. PloS One, 6(6), e21540. doi: 10.1371/journal. pone.0021540

- Cerqueira, E. M., Gomes-Filho I. S., Trindade, S., Lopes M. A., Passos, J. S., \& Machado-Santelli, G. M. (2004). Genetic damage in exfoliated cells from oral mucosa of individuals exposed to X-rays during panoramic dental radiographies. Mutation Research, 562(1-2), 111-117.
CONCLUSIONES

La revisión en la literatura del estado actual de la radiación ionizante $y$, de forma precisa, la referente a la radiación dental con rayos $X$, pone de manifiesto que:

- El potencial de daño celular debido a la exposición de energía radiante por rayos $X$ en odontología existe y es inevitable.

- El diagnóstico por imagen, sin duda invaluable, debe ejercerse de manera controlada. La radiación por rayos $X$ puede disminuirse a niveles realmente bajos mediante la tecnología digital para corrección de imagen. Por último, las normas gubernamentales sobre la radiación por rayos $X$ en constante revisión deberán apegarse a nuevas directrices para el control de radiación en el contexto internacional mediante tecnología, técnica radiográfica y conocimiento del cirujano dentista.
- Cho, Y. H., Kim, Y. J., An, Y. S., Woo, H. D., Choi, S. Y., Kang, C. M., \& Chung, H. W. (2009). Micronucleus-centromere assay and DNA repair gene polymorphism in lymphocytes of industrial radiographers. Mutation Research, 680(1-2), 17-24.

- Cmielova, J., Havelek, R., Kohlerova, R., Soukup, T., Bruckova, L., Suchanek, J., ..., Rezacova, M. (2013). The effect of ATM kinase inhibition on the initial response of human dental pulp and periodontal ligament mesenchymal stem cells to ionizing radiation. International Journal of Radiation Biology, 89(7), 501-511.

- Fung, H., \& Weinstock, D. M. (2011). Repair at single targeted DNA double-strand breaks in pluripotent and differentiated human cells. PLoS One, 6(5), e20514. doi: 10.1371/journal. pone.0020514

- Hart, D., Hillier, M. C., \& Wall, B. F. (2009). National reference doses for common radiographic, fluoroscopic and dental X-ray examinations in the UK. The British Journal of Radiology, $82(973), 1-12$.

- Hall, E. J., \& Giaccia, A. J. (2006). Radiobiology for the radiologist (6a. ed.). Philadelphia: Lippincott Williams \& Wilkins.

- Hayflick, L., \& Moorhead, P. S. (1961). The serial cultivation of human diploid cell strains. Experimental Cell Research, 25(3), 585-621.

- Hellén-Halme, K., \& Nilsson, M. (2013). The effects on absorbed dose distribution in intraoral $\mathrm{X}$-ray imaging when using tube voltages of 60 and $70 \mathrm{kV}$ for bitewing imaging. Journal of Oral and Maxillofacial Research, 4(3), e2. doi: 10.5037/ jomr.2013.4302 
- Henriques, S. (2013). A new way of thinking about patient radiation exposure [Informe electrónico]. Vienna: International Atomic Energy Agency.

- Holthausen, J. T., Wyman, C., \& Kanaar, R. (2010). Regulation of DNA strand exchange in homologous recombination. DNA Repair, 9(12), 1264-1272.

- International Atomic Energy Agency. (2013). X-rays-what patients need to know [Página informativa]. Recuperada de https://www.iaea.org/resources/rpop/patients-and-public/xrays

- Jacobson, L. O. (1952). Evidence for a humoral factor (or factors) concerned in recovery from radiation injury: A review. Cancer Research, 12(5), 315-325.

- Jiricny, J. (2006). The multifaceted mismatch-repair system. Nature Reviews Molecular Cell Biology, 7(5), 335-346.

- Kass, E. M., \& Jasin, M. (2010). Collaboration and competition between DNA double-strand break repair pathways. FEBS Letters, 584(17), 3703-3708.

- Kim, I. H., \& Mupparapu, M. (2009). Dental radiographic guidelines: A review. Quintessence International (Berlin, Germany: 1985), 40(5), 389-398.

- Kohn, M. M., Moores, B. M., Schibilla, H., Schneider, K., Stender, H. St., Stieve, F. E., ..., \& Wall, B. (Eds.). (1996). European guidelines on quality criteria for diagnostic radiographic images in paediatrics. Luxembourg: European Comission.

- Lee, C., Lee, S. S., Kim, J. E., Symkhampha, K., Lee, W. J., Huh, K. H., ..., Yeom, H. Y. (2016). A dose monitoring system for dental radiography. Imaging Science in Dentistry, 46(2), 103-108.

- Loubele, M., Bogaerts, R., Van Dijck, E., Pauwels, R., Vanheusden, S., Suetens, P., ..., Jacobs, R. (2009). Comparison between effective radiation dose of CBCT and MSCT scanners for dentomaxillofacial applications. European Journal of Radiology, 71 (3), 461-468.

- Luo, L. Z., Gopalakrishna-Pillai, S., Nay, S. L., Park, S. W., Bates, S. E., Zeng, X., ..., O'Connor, T. R. (2012). DNA repair in human pluripotent stem cells is distinct from that in non-pluripotent human cells. PLoS One, 7(3), e30541. doi: 10.1371/journal. pone.0030541

- Maynard, S., Swistowska, A. M., Lee, J. W., Liu, Y., Liu, S. T., Da Cruz, A. B., ..., Bohr, V. A. (2008). Human embryonic stem cells have enhanced repair of multiple forms of DNA damage. Stem Cells, 26(9), 2266-2274.

- Medina Gironzini, E. (2 de agosto de 2014). Nuevas tecnologías en el tratamiento de pacientes con cáncer en radioterapia y sus beneficios-B García [Serie de diapositivas en electrónico]. Recuperada de https://www.slideshare.net/medinao/ nuevas-tecnologias-en-el-tratamiento-de-pacientes-concancer-en-radioterapia-y-sus-beneficios-b-garcia

- Memon, A., Godward, S., Williams, D., Siddique, I., \& Al-Saleh, K. (2010). Dental X-rays and the risk of thyroid cancer: A casecontrol study. Acta Oncologica, 49(4): 447-453.
- Organización Mundial de la Salud. (s. f.). [Imagen]. Recuperada de www.who.int/es/

- Praveen, B. N., Shubhasini, A. R., Bhanushree, R., Sumsum, P. S., \& Sushma, C. N. (2013). Radiation in dental practice: Awareness, protection and recommendations. The Journal of Contemporary Dental Practice, 14(1), 143-148.

- Rehani \& Berris, T. (2013). Radiation exposure tracking: Survey of unique patient identification number in 40 countries. AJR American Journal of Roentgenology, 200(4), 776-779.

- Roberts, J. A., Drage, N. A., Davies, J., \& Thomas, D. W. (2009). Effective dose from cone beam CT examinations in dentistry. The British Journal of Radiology, 82 (973), 35-40.

- SEDENTEXCT Guideline Development Panel. (2012). Cone beam CT for dental and maxillofacial radiology. Radiation protection no. 172 [Reporte oficial]. Luxembourg: European Commission Directorate-General for Energy. Recuperado de http://ec.europa.eu/energy/nuclear/radiation_protection/ doc/publication/172.pdf

- Shao, L., Luo, Y., \& Zhou, D. (2014). Hematopoietic stem cell injury induced by ionizing radiation. Antioxidants \& Redox Signaling, 20(9), 1447-1462.

- Shin, H. S., Nam K. C., Park, H., Choi, H. U., Kim, H. Y., \& Park, C. S. (2014). Effective doses from panoramic radiography and $\mathrm{CBCT}$ (cone beam CT) using dose area product (DAP) in dentistry. Dento maxillo facial Radiology, 43(5), 20130439. doi: $10.1259 / \mathrm{dmfr} .20130439$

- Sonawane, A. U., Sunil Kumar, J. V., Singh, M., \& Pradhan A. S. (2011). Suggested diagnostic reference levels for pediatric X-ray examinations in India. Radiation Protection Dosimetry, 147(3), 423-428.

- Svilar, D., Goellner, E. M., Almeida, K. H., \& Sobol, R. W. (2011). Base excision repair and lesion-dependent subpathways for repair of oxidative DNA damage. Antioxidants \& Redox Signaling, 14(12), 2491-2507.

- Vermeulen, W. (2011). Dynamics of mammalian NER proteins. DNA Repair, 10(7), 760-771.

- Waingade, M., \& Medikeri, R. S. (2012). Analysis of micronuclei in buccal epithelial cells in patients subjected to panoramic radiography. Indian Journal of Dental Research: Official Publication of Indian Society for Dental Research, 23(5), 574578.

- White, S. C., \& Pharoah, M. J. (2004). Oral radiology: Principles and interpretration (6 $6^{a}$. ed.). St. Louis, MO: Elsevier.

- White, S. C., \& Mallya, S. M. (2012). Update on the biological effects of ionizing radiation, relative dose factors and radiation hygiene. Australian Dental Journal, 57(Suppl. 1), 2-8.

- Wilson, D. M., Kim, D., Berquist, B. R., \& Sigurdson, A. J. (2011). Variation in base excision repair capacity. Mutation Research, $711(1-2), 100-112$. 